\title{
All that glitters is not gold: sacroiliitis
}

\author{
Alfredo Tarantino, Justyna Paulina Jablonska, Paola D’Aprile \\ Department of Radiology, San Paolo Hospital, Bari, Italy
}

\begin{abstract}
Objectives: The aim of this study was to determine the prevalence of „rheumatic” and „non-rheumatic" changes of the sacro-iliac joints (SIJ).

Material and methods: We performed MRI in 210 patients with suspected inflammatory low back pain. We sorted and analysed the characteristics of sacroiliac bone lesions in „rheumatic” and „non-rheumatic” patients and assessed the diagnostic values of their extent and location. SIJ lesions were classified on the basis of their location into two categories: unilateral and bilateral. Their extent was then measured and assigned to one of two groups: $<1 \mathrm{~cm}$ or $\geq 1 \mathrm{~cm}$.

Results: In 45 cases (21\%), the MRI findings matched the clinical diagnosis of „rheumatic” sacroiliitis. Interestingly, in 99 cases (47\%) the SIJ changes were classified as „,non-rheumatic”. L5-S1 degenerative changes, scoliosis and pelvic asymmetry were most frequently encountered as concomitant phenomena in our study.

Conclusions: MRI of the sacroiliac joints in patients suspected of inflammatory low back pain demonstrated more often "non-rheumatic" changes.
\end{abstract}

Key words: rheumatic diseases, magnetic resonance imaging, sacroiliitis.

\section{Introduction}

Low back pain (LBP) is second only to the common cold as a cause of medical visits to general practitioners. Approximately $90 \%$ of adults experience back pain at some point in their lives [1]. At the beginning of the $20^{\text {th }}$ century, the sacro-iliac joints (SIJ) were considered the most important source of low back pain. Over the past three decades, with the increased tendency to diagnose lumbar disc herniation as a cause of low back pain, the role of SIJ in the genesis of this complaint has decreased in importance [2].

The term sacroiliitis is generally used to indicate inflammation with oedema of the SIJ of so-called "rheumatic" origin (i.e. indicating seronegative spondyloarthritis), but similar oedematous lesions may be of "non-rheumatic" origin (e.g. with degenerative or infectious pathogenesis), in patients with low back pain. Distinguishing "rheumatic" from "non-rheumatic" sacroiliitis is very important in terms of therapeutic choice, sometimes very expensive [3].
The Assessment of SpondyloArthritis International Society (ASAS) considers magnetic resonance imaging (MRI) positive for sacroiliitis when the following criteria are fulfilled: "Bone marrow oedema (BMO) is depicted as high signal on STIR (short TI inversion recovery) or T2-weighted fat-saturated (T2FS) images, typically located periarticularly. Bone marrow oedema is highly suggestive of sacroiliitis when clearly present and located in the typical anatomical areas (subchondral or periarticular bone marrow). If there is only one lesion per MRI slice suggesting active inflammation, the lesion should be present on at least two consecutive slices. If there is more than one lesion on a single slice, one slice may be sufficient" [4].

The aim of this study was to determine the prevalence of "rheumatic" and "non-rheumatic" changes of the SIJ demonstrated by MRI in patients with chronic low back pain suspected of sacroiliitis. We analysed MR characteristics of the bone marrow oedema of "rheumatic" and "non rheumatic" origin, trying to assess 
the diagnostic values of its extent and location (uni- or bilateral).

\section{Material and methods}

This study was approved by the institutional ethics committee with number 5607, prot. 0046718/01/06/2018. Informed consent was waived as it was a retrospective study.

All patients recruited for this study were outpatients referred for MRI to our Neuroradiology Department with chronic low back pain (> 3 months) suspected of sacroiliitis, in which characteristics of pain were mixed (inflammatory and non-inflammatory). All 210 patients - 135 women (64\%) and 75 men (36\%), with a mean age of 44 years (range 18-60) - met the inclusion criteria.

MRI was performed on a $1.5 \mathrm{~T}$ MRI unit (Siemens, Simphony TIM). The study protocol included: axial SE T1-weighted images (slice thickness $3.5 \mathrm{~mm}$ ), axial and coronal TSE T2-weighted images with fat saturation (slice thickness $4 \mathrm{~mm}$ ), oriented along the axis of the sacral bone.

The SIJ MRI examinations were read independently by experienced board certified neuroradiologists. The evaluation procedure was precisely defined and explained in the study protocols. For practical purposes and to facilitate the diagnosis, SIJ oedematous lesions (high signal areas in T2-weighted images with fat saturation) were classified on the basis of their location into two categories: unilateral and bilateral. Their extent was measured through the major diameter and assigned to one of two groups: $<1 \mathrm{~cm}$ (small) or $\geq 1 \mathrm{~cm}$ (large).

Non-inflammatory concomitant conditions were evaluated to be present or absent: L5-S1 degenerative changes, lumbosacral transitional anomaly, pelvic asymmetry, fracture, tumour, infection.

Official reports were used to generate data in this study. All the patients were afterwards clinically fully assessed by a consultant rheumatologist. The rheumato-
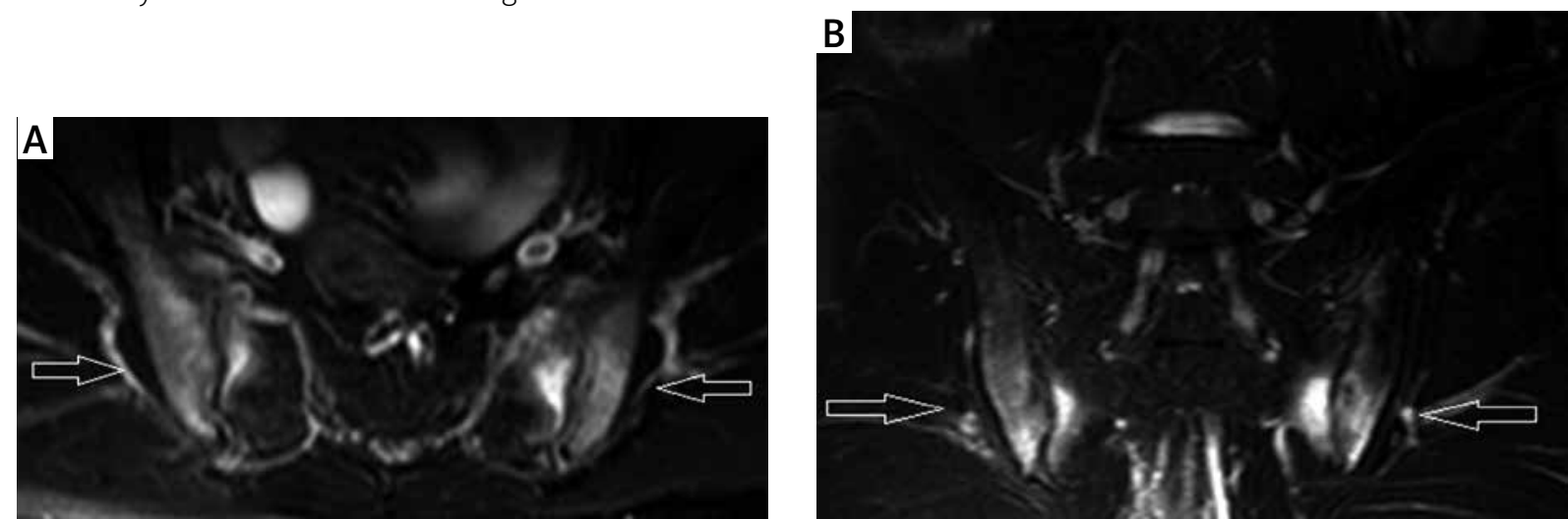

Fig. 1. Sacro-iliac joints MRI. Axial (A) and coronal (B) TSE T2W images with fat saturation. Bilateral subchondral bone oedema $\geq 1 \mathrm{~cm}$ (arrows). "Rheumatic" sacroiliitis (with inflammatory pathogenesis). 
The probability that bilateral lesions $\geq 1 \mathrm{~cm}$ are rheumatic is 99\% higher than $<1 \mathrm{~cm}$ lesions. The probability that unilateral lesions $\geq 1 \mathrm{~cm}$ are not rheumatic is 24 times higher than symmetric lesions (Table III).

Small unilateral lesions $<1 \mathrm{~cm}$ have the likelihood of being non-rheumatic of $95 \%$. The probability that bilateral lesions $\geq 1 \mathrm{~cm}$ are not rheumatic is only $8 \%$.

\section{Discussion}

Bone marrow oedema of the SIJ is not a specific finding for the so-called "rheumatic" sacroiliitis (i.e. sacroiliitis in spondyloarthritis, with immunological inflammation [5]) and may also be seen in degenerative disease (i.e. degenerative-mechanical based inflammation). In particular, sacroiliac arthrosis may present an inflammatory oedematous component. Furthermore, noninflammatory diseases such as L5-S1 degenerative changes, lumbosacral transitional anomaly, pelvic asymmetry, fracture, tumour, and infection may clinically present as inflammatory low back pain $[6,7]$.

MRI has made a major contribution in the last decade to a better understanding of SIJ disease. In particular, MRI emerges as the gold standard technique to an early diagnosis of BMO. It is mandatory to use a proper MRI study protocol, including fat-suppressed T2-weighted images, in order to clearly visualize hyperintensity corresponding to oedematous lesions [8].

For decades many authors have always been concerned with SIJ lesions in an attempt to give a definite aetiopathogenesis to the bone changes. Currently SI disease is a topic intensively discussed in the literature due to the scientific studies that report major prevalence of non-rheumatic origin of the alterations [9]. Degenerative pathology of SIJ is an underestimated condition and has remained largely outside the research spotlight. This lack of attention leads to overdiagnosis of rheumatic sacroiliitis in patients with only isolated bone oedema in MRI.

Musculoskeletal disorders are the main cause of morbidity in the world. These pathologies have a substantial impact on health and quality of life and result in huge costs for healthcare systems, according to the World Health Organization. Although low back pain is a benign medical problem, it is responsible for direct

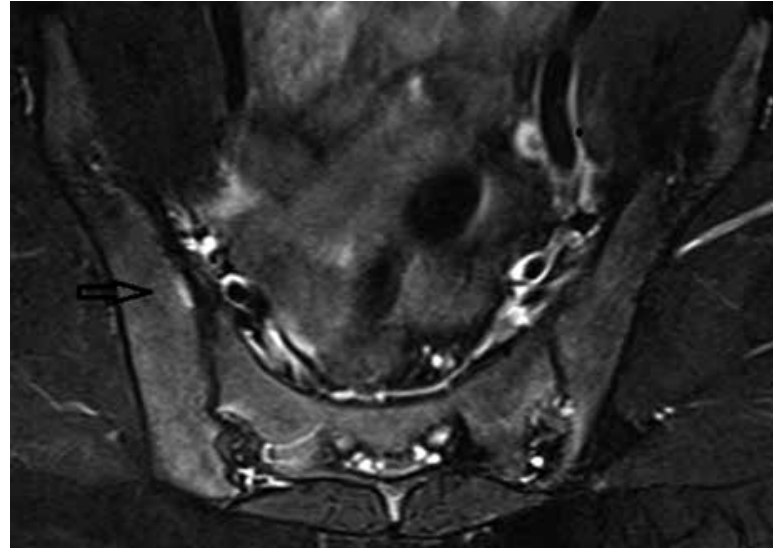

Fig. 2. Sacro-iliac joints MRI. Axial (A), coronal (B) and sagittal (C) TSE T2W images with fat saturation. Unilateral subchondral bone oedema $<1 \mathrm{~cm}$ (arrow). Associated anterolisthesis of L4 over L5. "Non-rheumatic" sacroiliitis (with degenerative-mechanical pathogenesis).

care expenditures ranging from $\$ 5$ billion [10] to more than $\$ 20$ billion annually and as much as $\$ 50$ billion a year if indirect costs are included [11]. In any 12-month period, $7 \%$ of adults consult for this complaint [12]. In particular, overdiagnosis of sacroiliitis may result in the inappropriate prescription of treatments that cost more than 1000 euro per month yet are not more effective than placebo [13].

In the light of the experience derived from so many cases we have been able to observe in our department, we also decided to contribute to the research by con-

Table I. Sacro-iliac joints changes distribution

\begin{tabular}{lccc}
\hline \multirow{2}{*}{ Aetiopathogenesis } & Location & \multicolumn{2}{c}{ Extent } \\
\cline { 3 - 4 } & & $<1 \mathrm{~cm}$ & $\geq 1 \mathrm{~cm}$ \\
\hline Rheumatic & Bilateral & 3 & 36 \\
\hline Rheumatic & Monolateral & 3 & 3 \\
\hline Total & & 6 & 39 \\
\hline Non-rheumatic & Bilateral & 30 & 3 \\
\hline Non-rheumatic & Monolateral & 60 & 6 \\
\hline Total & & 90 & 9 \\
\hline Total & & 96 & 48 \\
\hline
\end{tabular}

Table II. Results of statistical analysis

\begin{tabular}{|c|c|c|c|c|c|}
\hline Effect & $\mathrm{B}$ & SE & $\chi^{2}$ wald & $\mathrm{df}$ & $p$ \\
\hline Distribution & 0.69 & 0.85 & 0.67 & 1 & 0.413 \\
\hline Extent & -4.79 & 0.85 & 31.49 & 1 & $<0.001$ \\
\hline Correlation & 2.49 & 1.26 & 3.91 & 1 & 0.048 \\
\hline Constant value & 2.30 & 0.61 & 14.46 & 1 & $<0.001$ \\
\hline
\end{tabular}


Table III. Odds ratios

\begin{tabular}{lcc}
\hline Lesion property & $\begin{array}{c}\text { Radiological evaluation } \\
\text { result }\end{array}$ & $\begin{array}{c}\text { Odds } \\
\text { ratio }\end{array}$ \\
\hline Distribution & Monolateral & 0.100 \\
\hline Bilateral & 0.008 \\
\hline$\geq 1$ & 23.999 \\
\hline
\end{tabular}

ducting this study and proposing this simple evaluation system of the SIJ.

The differential diagnosis between rheumatic and non-rheumatic changes of the SIJ is determinant because the therapeutic treatment is different in these two conditions. In particular, only patients with rheumatic sacroiliitis need expensive medical therapies with biological medicines, clinical and MRI follow-up and may be subjects to frequent job absences, exposing the whole social system to enormous costs. In contrast, degenerative-mechanical alterations require only, if really necessary, medical therapy and physiotherapy for osteoarthrosis.

Our study shows that in patients with suspected inflammatory low back pain, MRI SIJ evaluation provided more often nonrheumatic lesions than rheumatic. Degenerative bone changes were about two times more common than inflammatory. Degenerative SIJ disease may be present in patients younger than 40 years and is consistently present in those over 50 years of age [14].

L5-S1 degenerative changes, scoliosis and pelvic asymmetry were most frequently encountered as concomitant phenomena in our study. Degenerative phenomena of the lumbar spine may be present in up to $40 \%$ of subjects under the age of 30 years. Lumbosacral transition anomaly is present in at least $4 \%$ of the population [15]. Fractures are found in 1-5\% of the population at risk (osteoporosis, osteopenia, post-actinic bone weakening) [16]. No tumours, fractures or infections were found in our patients. This may be in part due to the distinct clinical presentation and, in part, because of low incidence of these entities. However, it is advised that these alterations with non-rheumatic aetiopathogenesis should be carefully assessed and recognized.

Moreover, in our study $32 \%$ of SIJ MRI examinations were completely negative.

Currently applied clinical criteria for inflammatory back pain include: age at onset $<40$ years, insidious onset, improvement with exercise, no improvement with rest and pain at night. Even if 4 of 5 criteria are present, the specificity for diagnosis of inflammatory disease is no more than $72 \%$ [4].
Our method of SIJ MRI evaluation in patients with suspected inflammatory low back pain has specificity of $97 \%$, and that is of great diagnostic value. In addition, the concomitant presence of non-inflammatory bone changes can be very helpful in the correct diagnosis of aetiology of a sacroiliac joint alteration.

\section{Conclusions}

Some conclusions can be drawn from our findings. MRI of the sacroiliac joints in patients suspected of inflammatory low back pain demonstrated more often non-rheumatic changes.

The principles of diagnostic imaging that we suggest adopting consist in a simple evaluation of SIJ lesions: identify the oedematous changes, determine their location within the joints (bilateral or unilateral), define their extension and evaluate concomitant phenomena (L5-S1 disc degenerative changes, scoliosis and pelvic asymmetry), as quickly, efficiently and cost-effectively as possible.

The authors declare no conflict of interest.

\section{References}

1. Frymoyer JW. Back pain and sciatica. N Engl J Med 1988; 318 : 291-300.

2. Fortin JD, Dwyer AP, West S. Sacroiliac joint: pain referal maps upon applying a new injection/arthrography technique. Part I: Asymptomatic volunteers. Spine 1994; 19: 1475-1482.

3. Tarantino A, Jablonska JP, D'Aprile P. What's in a name? That which we call sacroiliitis by any other name would look the same... Reumatologia 2018; 56: 129-130.

4. Sieper J, van der Heijde D, Landewé R, et al. New criteria for inflammatory backpain in patients with chronic back pain: a real patient exercise by experts from the Assessment of SpondyloArthritis international Society (ASAS). Ann Rheum Dis 2009; 68: 784-788.

5. Generali E, Bose T, Selmi C, et al. Classification of spondyloarthritis as autoimmune or autoinflammatory. Autoimmun Rev 2018; 17: 935-941.

6. Bereau M, Prati C, Wendling D. Sacroiliac edema by MRI does not always indicate spondylarthritis. Joint Bone Spine 2011; 78: 646.

7. Schueller-Wiedekamm C, Schueller G. Sacroiliitis oder pseudosacroiliitis. Radiologe 2012; 52: 132-140.

8. D'Aprile P, Tarantino A. MRI of rheumatic spine. A case based atlas. Springer 2014.

9. Berthelot JM, le Goff B, Maugars Y, Laredo JD. Sacroiliac joint edema by MRI: far more often mechanical than inflammatory? Joint Bone Spine 2016; 83: 3-5.

10. Drezner JA, Herring SA. Managing low-back pain. Steps to optimize function and hasten return to activity. Phys Sportsmed 2001; 29: 37-43. 
11. Deyo RA, Chrkin D, Conrad D. Cost, controversy, crisis: low back pain and the health of the public. Annu Rev Public Health 1991; 12: 141-156.

12. McCormick A, Fleming D, Charlton J. Morbidity statistics from general practice. Fourth national study 1991-1992. HMSO Office of Population Censuses and Surveys 1995; Series MB5 No 3, London.

13. Claudepierre P, Wendling D, Breban M. Ankylosing spondylitis, spondyloarthropathy, spondyloarthritis or spondylarthritis: what's in a name? Joint Bone Spine 2012; 79: 534-535.
14. Shibata Y, Shirai Y, Miyamoto M. The aging process in the sacroiliac joint: helical computed tomography analysis. J Ortop Sci 2002; 7: 12-18.

15. Konin JP, Walz DM. Lumbosacral transitional vertebrae: classification, imaging, findings and clinical relevance. Am J Neuroradiol 2010; 31: 1778-1786.

16. Lyders EM, Whitlow CT, Baker MD, et al. Imaging and treatment of sacral insufficiency fractures. Am J Neuroradiol 2010; 31: 201-210. 